\title{
Lung carcinomas with a basaloid pattern: a study of 90 cases focusing on their
} poor prognosis

\author{
D. Moro-Sibilot*, , S. Lantuejoul**, S. Diab\#, N. Moulai", A. Aubert*,+, J.F. Timsit*,\#, \\ C. Brambilla*,\#, P.Y. Brichon*, and E. Brambilla*,
}

ABSTRACT: Lung carcinoma with a basaloid pattern $(\mathrm{BC})$ is classified as either a basaloid variant of squamous cell carcinoma (SCC) or as variant of large cell carcinoma (LCC) depending on the presence of a squamous component. In a previous study of 37 cases, the present authors showed that BC presented with a shorter median and overall survival.

In order to confirm its clinical significance in a larger series, 90 BC, including 46 basaloid variants of LCC and 44 basaloid variants of SCC, were compared with 1,328 other nonsmall cell lung carcinoma (NSCLC) with regard to clinical features and survival.

The survival of basaloid variants of LCC and SCC was comparable. Median and overall survival were significantly lower for BC than for NSCLC in stage I-II patients, with a median survival of 29 and 49 months, respectively, and 5-yr survival rates of 27 and $44 \%$ for BC and NSCLC. When disease-specific survival was considered, BC had a shorter survival than both NSCLC and SCC.

Basaloid pattern confers a poor prognosis in nonsmall cell lung carcinoma, especially in stage I-II patients, suggesting that lung carcinoma with a basaloid pattern is not only a variant of squamous cell carcinoma or large cell carcinoma, but is a unique entity with a significantly poor prognosis.

KEYWORDS: Basaloid carcinoma, diagnosis and staging, lung cancer, pathology

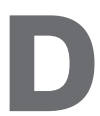

uring the last 20 yrs, basaloid carcinomas have been identified in organs other than the lungs as aggressive tumours with a poor prognosis [1-7]. In 1992, the current authors first reported this entity in the lung and described its clinicopathological features in a founding paper based on the observation of 37 basaloid carcinomas [1]. Carcinoma with basaloid pattern is now recorded in the World Health Organization (WHO) classification either as a variant of large cell carcinoma (LCC) in its pure form, or as a basaloid variant of squamous cell carcinoma (SCC) when associated with areas of squamous differentiation. Interestingly, these two histological subtypes, which both belong to the nonsmall cell lung carcinoma (NSCLC) group, share a poor prognosis when compared with other NSCLCs, with a median survival of 20 months in the operable stages [1, 7-9]. This finding led to their recent inclusion in the revised WHO classification alongside other prognostic entities, such as large cell neuroendocrine (NE) carcinomas or sarcomatoid carcinomas [10-15].
However, their prognostic significance has recently been questioned in a report based on a series of 35 basaloid carcinomas [16], which prompted the current authors to carry out a new analysis on a larger series of 90 cases with a longer follow-up. The previous observations are confirmed herein, and it is demonstrated that the basaloid pattern confers in itself a poor prognosis irrespective of the variant it belongs to, justifying the inclusion of the two lung carcinoma variants with a basaloid pattern within the same clinicopathological entity.

\section{MATERIALS AND METHODS \\ Patients}

Between January 1, 1979 and December 31, 2003, 90 out of 1,418 cases of NSCLCs were classified according to the revised WHO classification criteria $[10,11]$ either as a basaloid variant of LCC $(n=46)$ or as a variant of SCC $(n=44)$ by two expert thoracic pathologists (E. Brambilla and S. Lantuejoul). Among them, 37 cases have already been described in the founding paper in 1992. In

\section{AFFLLIATIONS}

*Lung Cancer Research Group, Institut National de la Santé et de la Recherche Médicale, Institut A. Bonniot, La Tronche,

\#Pole de Médecine Aigue Communautaire, Pneumologie,

'Département d'Anatomie et Cytologie Pathologiques, and +Département de Chirurgie Thoracique, Cardiovasculaire et Endocrinienne Hôpital Albert Michalon, Grenoble, France.

CORRESPONDENCE

D. Moro-Sibilot

DMAS Pneumologie

Hôpital Albert Michalon BP217 38043 Grenoble Cedex 9

France

Fax: 33476765617

E-mail: DMoro.pneumo@

chu-grenoble.fr

Received:

May 142007

Accepted after revision:

December 032007

SUPPORT STATEMENT

The study recieved financial support from INSERM, Institut National du Cancer PNES2006, Centre de ressources biologiques 2003-2004, EU Grant QLG1-CT 2002-01735.

STATEMENT OF INTEREST

None declared. 
the current study, the authors decided to analyse all carcinomas with basaloid pattern (BC group) within the same group. The group of 1,328 patients with lung carcinomas without a basaloid pattern (non-BC group) included other histological subtypes, all without basaloid features (i.e. SCC, $n=884$; adenocarcinomas, $n=387 ; \mathrm{LCC}, \mathrm{n}=57$ ). Large cell NE carcinomas were not included in the control group due to previously established unfavourable prognosis. All cases were resected in the same institution. The post-operative stage distribution was as follows: stage I, 582 patients (BC 38, nonBC 544); stage II, 459 patients (BC 28, non-BC 431); stage IIIA, 369 patients (BC 16, non-BC 353); and stage IIIB/IV, eight BC patients. The main clinical and surgical characteristics are detailed in table 1.

\section{Pathological examination}

The basaloid pattern was characterised according to the 1999 and $2004 \mathrm{WHO}$ classifications by a proliferation of relatively small cells presenting a typical high mitotic rate, forming a lobular pattern with peripheral palisading and comedo-type necrosis $[10,11]$. The basaloid variant of LCC was considered when cells were devoid of intercellular bridges or individual cell keratinisation. However, considering the first description of basaloid carcinoma by BRAMBILLA et al. [1], it was decided to include the presence of foci of abrupt pearl formation in the absence of progressive squamous maturation as an additional criterion. In contrast, the variant of SCC was retained when squamous differentiation was obvious, but represented $<50 \%$ of the tumour, which is in agreement with the 1999 classification. As $30 \%$ of basaloid carcinomas can exhibit rosettes, the absence of immunohistochemical NE markers was systematically provided to rule out large cell NE carcinomas or small cell lung carcinomas. In addition, since the basaloid pattern, as in any nonsmall cell carcinoma, may display one or two NE markers in a small proportion $(<10 \%)$ of tumour cells, the diagnosis of carcinomas with a basaloid pattern was maintained when $<10 \%$ cells displayed one NE marker in the presence of the expression of a specific set of cytokeratins (CKs; 1, 5, 10 and 14) recognised by the $34 \beta E 12$ monoclonal antibody; these CKs are never expressed in NE tumours [8]. The post-surgical (pathological) stages were determined according to the international Tumor, Node, Metastases (TNM) American Joint Committee on Cancer Union (AJCC)/Union Internationale Contre le Cancer (UICC) classification [17].

\section{Data collection}

All surgical resections have been prospectively registered since 1998 in a computerised database. Cases prior to 1988 were revisited before registration, and all cases were reclassified using the TNM AJCC/UICC classification [17]. As the median age of all patients was $63 \mathrm{yrs}$, two age categories, $\leqslant 63 \mathrm{yrs}$ and $>63 \mathrm{yrs}$, were considered in univariate and multivariate analyses. The Charlson comorbidity index was computed for each patient [18].

\section{Statistical analysis}

Descriptive analyses comparing categorical variables were carried out using Fisher's exact tests. Descriptive analyses comparing continuous and two-level categorical variables were carried out using unpaired t-tests. Median follow-up was calculated using the method of SCHEMPER and SMITH [19]. Overall survival was calculated from the date of surgery to the last day of follow-up or death. Disease-specific survival was computed using only lung cancer deaths and censoring all other causes of death. All patients surviving $>5 \mathrm{yrs}$ were censored after this time in order to avoid bias due to late mortality associated with comorbidity. Deaths occurring during the first 30 days after surgery were considered as hospital mortalities. Univariate survival analyses were performed using the Kaplan-Meier method and the log-rank test.

\section{TABLE 1 Clinical and demographic characteristics of the different groups of patients}

\begin{tabular}{|c|c|c|c|c|c|}
\hline Variables & $\mathrm{BC}$ & Non-BC & $\mathrm{p}$-value $\mathrm{BC} /$ non-BC & scc & $\mathrm{p}$-value $\mathrm{BC} / \mathrm{SCC}$ \\
\hline Subjects & 90 & 1328 & & 884 & \\
\hline Age yrs & $63.6(37-82)$ & $62.4(31-86)$ & NS & $62.9(31-86)$ & NS \\
\hline Tobacco use mean pack-yrs & 46.0 & 32 & $<0.0001$ & 33.5 & 0.0002 \\
\hline \multicolumn{6}{|l|}{ Post-operative stage } \\
\hline IIIA & 16 & 353 & & 224 & \\
\hline IIIB, IV & 8 & 0 & & 0 & \\
\hline No & 42 & 658 & 0.03 & 407 & NS \\
\hline N1 & 40 & 444 & & 350 & \\
\hline N2 & 8 & 226 & & 127 & \\
\hline Pneumonectomy & 22 & 320 & NS & 587 & NS \\
\hline
\end{tabular}

Data are presented as $\mathrm{n}$ and mean (range), unless otherwise indicated. BC: lung carcinomas with a basaloid pattern; non-BC: nonbasaloid carcinoma; SCC: squamous cell carcinoma; N0, N1, N2: three of the four classes of N status in the international Tumor, Node, Metastases classification; ClS: carcinoma in situ; NS: nonsignificant. 
Multivariate analysis was performed using a Cox proportional hazard model. Hazard ratios and their 95\% confidence intervals were calculated. A p-value of $<0.05$ was considered statistically significant.

Survival analyses were performed on the entire cohort of patients. To avoid bias related to organ failure caused by tumour compression or involvement, survival analyses were performed for stage I patients and stage I and II patients. Moreover, to avoid all bias related to peri-operative mortality, analyses were carried out on patients surviving 1 month after the surgical resection. In order to obtain a sufficient number of patients and to avoid underpowered analyses, the latter survival analyses were performed on stage I and II patients (BC, 59 patients; others, 913 patients).

\section{RESULTS}

Among a cohort of 1,418 patients, 90 BC were diagnosed (prevalence $6.3 \%$ ). In the group of 90 carcinomas with basaloid pattern, 46 basaloid variants of LCCs and 44 basaloid variants of SCC were identified and considered as a whole (fig. 1). The clinical characteristics of BC and other NSCLCs without basaloid features (i.e. non-BC) are shown in tables 1 and 2 .

Most of the clinical characteristics were well balanced between these two groups. Patients with BC had greater tobacco consumption than patients with non-BC. The presence of a single or multiple synchronous carcinoma in situ (CIS) on the surgical specimen was more frequent in the $\mathrm{BC}$ group than in the non-BC and SCC groups (table 1).

The median duration of follow-up was 109 months and the median actuarial survival of the 1,418 patients was 40 months. At the time of the current study, 972 patients died (BC, 70; non$B C, 902), 471$ of them with a relapse of lung cancer $(B C, 46$;

\begin{tabular}{lccc}
\hline TABLE 2 & $\begin{array}{l}\text { Clinical, demographic and survival } \\
\text { characteristics of the two groups of stage I } \\
\text { patients }\end{array}$ & Non-BC & p-value \\
\hline Variables & BC & 544 & \\
\hline Subjects & 38 & $488 / 56$ & NS \\
Males/females & $38 / 0$ & $62.4(33-86)$ & 0.03 \\
Age yrs & $65.8(37-79)$ & 305 & NS \\
Right-sided tumour & 19 & 47 & NS \\
Pneumonectomy & 4 & 459 & \\
Lobectomy & 30 & 38 & NS \\
Segmentectomy & 4 & 26 & NS \\
Post-operative deaths & 4 & 230 & \\
Post-operative stage & & 314 & NS \\
$\quad$ T1 & 22 & 497 & 0.01 \\
$\quad$ T2 & 16 & 51 & \\
Charlson index $\leqslant \mathbf{2}$ & 32 & Not reached at 5 yrs & \\
Median survival & 36 months & & \\
Survival $>\mathbf{5}$ yrs \% & 33 & &
\end{tabular}

Data are presented as $\mathrm{n}$ or mean (range), unless otherwise indicated. $\mathrm{BC}$ : lung carcinomas with a basaloid pattern; non-BC: nonbasaloid carcinoma; T1, T2: two of the four classes of $\mathrm{T}$ status in the international Tumor, Node, Metastases classification; NS: nonsignificant.
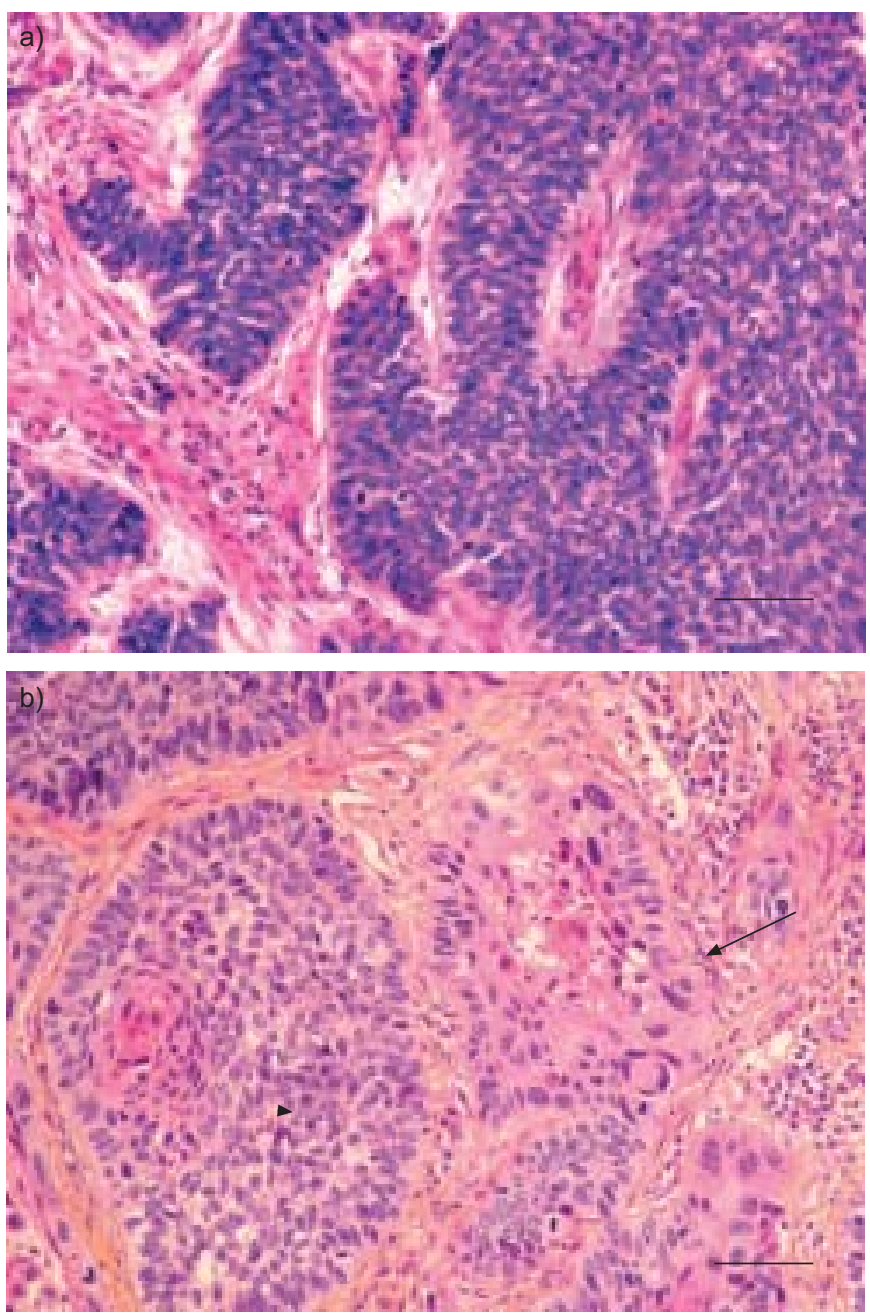

FIGURE 1. a) Basaloid variant of large cell carcinoma, composed of small-size cells, arranged in a typical lobular pattern with peripheral palisading; no squamous differentiation (haematoxylin-eosin-saffron stain). b) Basaloid variant of squamous cell carcinoma. The arrow shows a lobule exhibiting obvious squamous differentiation. The arrowhead shows a lobule with basaloid features (haematoxylin-eosin-saffron stain). Scale bars $=80 \mu \mathrm{m}$.

non-BC, 425). Stage I patients had comparable clinical and therapeutic characteristics within the $\mathrm{BC}$, non-BC and SCC groups (table 2); however, patients with $B C$ were slightly older than the non-BC patients (mean age 65.8 versus $62.4 \mathrm{yrs}$; $\mathrm{p}=0.03$ ). The causes of death for stage I patients are detailed in an additional table (see supplementary material).

No significant difference in survival between patients with a basaloid variant of LCCs and those with a basaloid variant of SCC was observed $(\log \operatorname{rank} p=0.34)$, although a trend for shorter survival could be noted in the basaloid variant of the LCC group (median survival 29 versus 36 months). Survival analyses performed in the different stages and histological groups are reported in table 3. Among these analyses, the most significant differences were observed in the cohort of early stage I and II patients (figs 2 and 3).

When disease-specific survival was considered, BC subjects had a shorter survival than non-BC (log rank $\mathrm{p}=0.014)$ and 
SCC (log rank $\mathrm{p}=0.005)$ subjects. Basaloid variants of SCC were compared with SCC in stage I patients. Despite median and 5-yr survival being very similar to those observed in other analyses at stage I, the log-rank test was not significant $(p=0.19)$, and this might be explained by the small number of patients showing stage I basaloid variants of the SCC group (19 patients).

Univariate analyses in stage I patients identified three other significant factors of prognosis: pathological T status $(p=0.01)$, age $\geqslant 63$ yrs $(p=0.0007)$ and a Charlson comorbidity index $>2$ $(\mathrm{p}=0.0007$; table 4$)$.

Cox proportional hazard models evaluating the independent hazard associated with baseline covariates were performed for stage I patients. Age, T status, presence of a basaloid pattern and a Charlson comorbidity index of $>2$ were associated with a prognostic significance (table 4 , model A). When excluding early post-operative deaths from the cohort of patients, age, T status, presence of a basaloid pattern and a Charlson comorbidity index of $>2$ were still associated with a prognostic significance (table 4 , model B).

\section{DISCUSSION}

$\mathrm{BC}$ is a rare histological subtype of lung cancer with a prevalence of $6.3 \%$ in the current study based on 1,418 NSCLC consecutive patients treated in the same institution. This type of tumour is well recognised in other locations, such as the head and neck, oesophagus and anal canal, where it is defined as "basaloid squamous cell carcinoma", irrespective of the amount of squamous differentiation. It has a peculiarly aggressive behaviour, characterised by a high frequency of lymph node and visceral metastases [1-6]. However, due to the distinction in the WHO classification of lung tumours of two basaloid variants belonging either to the SCC group or to the LCC group, diagnosis of lung carcinomas with a basaloid pattern remains a difficult area for pathologists. First, basaloid carcinoma pattern may represent diagnostic pitfalls, particularly on small or crushed tissue samples from bronchial or transthoracic fine needle biopsies [1, 11]. Secondly, it can be misdiagnosed either as adenoid cystic carcinoma, small cell lung carcinoma or large cell NE carcinoma; adenoid cystic

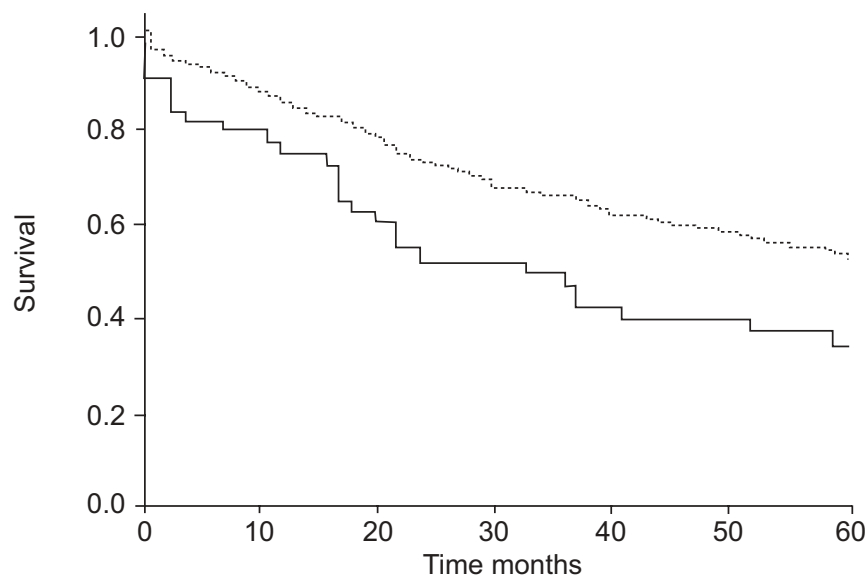

FIGURE 2. Overall survival curves for stage I patients. Lung carcinomas with a basaloid pattern (-) versus nonbasaloid carcinoma (-----). Log rank $p=0.01$

carcinoma typically lacks a high mitotic index and comedonecrosis. With the help of a newly described panel of immunohistochemical markers, small cell lung carcinoma and large cell NE carcinoma can be easily ruled out by the demonstration of CKs 1, 5, 10 and 14, and the lack of NE markers [8]. However, the diagnosis of poorly differentiated SCC is possibly the most difficult to exclude, as it is only based on negative morphological criteria, i.e. the absence of a basaloid pattern, which is difficult to ascertain on a biopsy specimen. In addition, the 1999 and 2004 WHO classifications are too restrictive for the diagnosis of basaloid variant of LCC; for instance, the presence of abrupt keratin pearl formation, mentioned in the very first description by BRAMBILLA et al. [1] in addition to absence of intercellular bridges and individual cell keratinisation, is no longer admitted in the WHO classification, which probably leads to the rarity of this diagnosis. On the contrary, histological recommendations are too vague for the diagnosis of a basaloid variant of SCC, retained when squamous differentiation is prominent but not predominant; conversely, it could be suspected that this variant is too frequently assimilated as a SCC. As a first step, the present authors therefore decided to include all

TABLE 3 Univariate survival analyses performed in the different groups and different histological subtypes

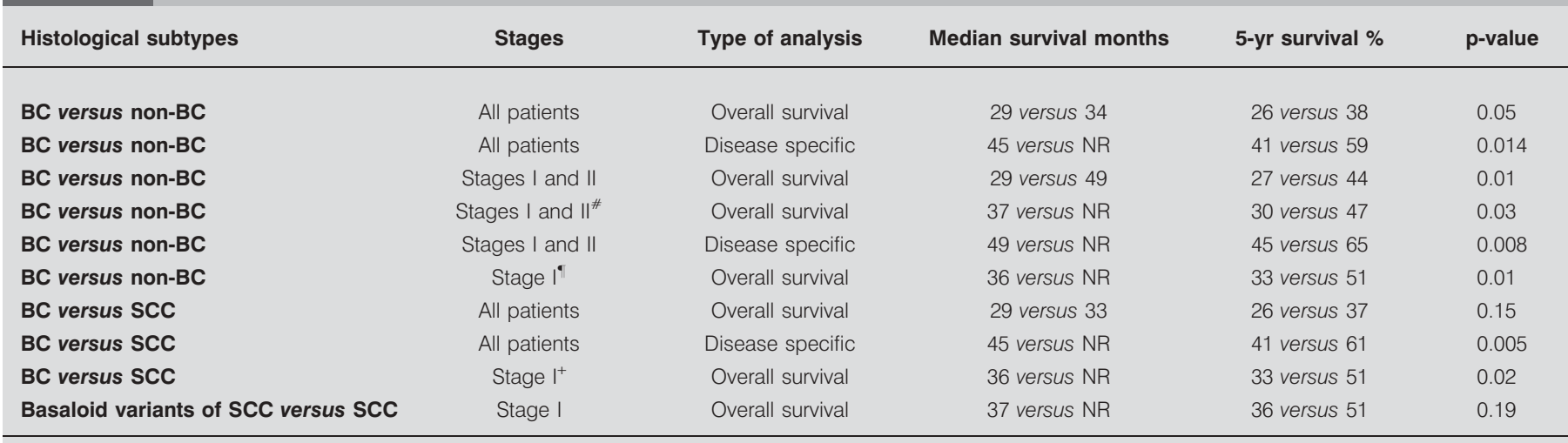

BC: lung carcinomas with a basaloid pattern; non-BC: nonbasaloid carcinoma; SCC: squamous cell carcinoma; NR: not reached. "\#: patients who died within 30 days after surgery were excluded; ${ }^{\circ}$ : see figure $2{ }^{+}$: see figure 3. 


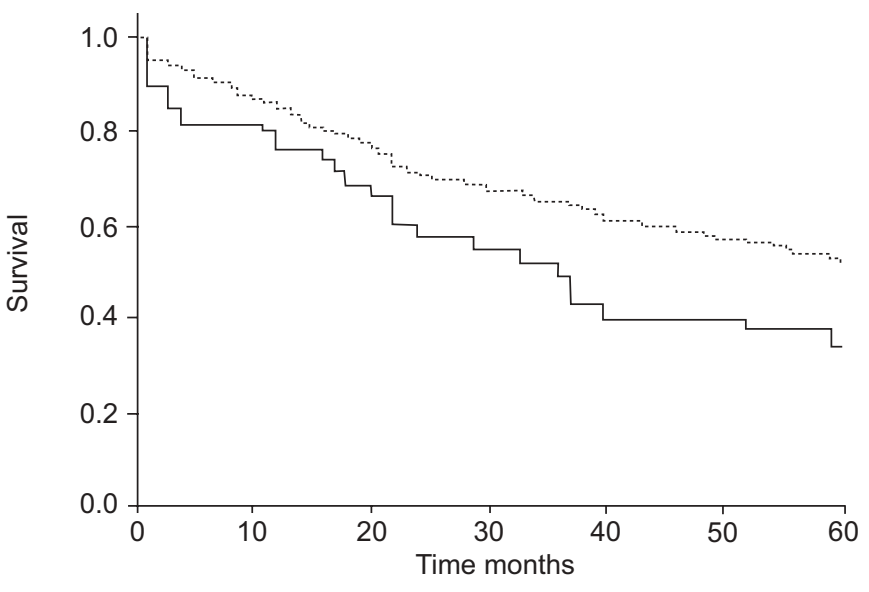

FIGURE 3. Overall survival curves for stage I patients. Lung carcinomas with a basaloid pattern (-) versus squamous cell carcinoma (-----). Log rank $\mathrm{p}=0.02$.

patients with basaloid features within the same histological class of $\mathrm{BC}$ from the current study in order to re-evaluate the prognostic significance of basaloid pattern in comparison with SCC and non-BC.

In the original publications in 1992 and 1994 [1, 7], a poorer prognosis of 37 lung carcinomas with a basaloid pattern of stages I and II was observed, as compared with 40 poorly differentiated SCC of the lung. However, a recent study published by KIM et al. [16], based on 291 surgically resected lung tumours including 167 poorly differentiated SCC and 35 basaloid carcinomas compared in terms of clinical data and survival outcome, questioned their prognostic significance. Although the overall prevalence of basaloid carcinomas was $4.8 \%$, consistent with data from the present study, even if slightly lower, the authors found no difference in survival and behaviour between BC and poorly differentiated SCC. However, as 14 of their 35 cases expressed at least one NE marker, two cases stained two NE markers, and one expressed synaptophysin but no CK 1, 5, 10 or 14, the series of KIM et al. [16] could be suspected of including some large cell NE carcinoma. The present series remains the largest one reported with the longest median follow-up $[7,16]$. Whereas the actuarial 5-yr survival rate of the present study's SCC group was equivalent to that observed by КIM et al. [16], being 38 versus $40.6 \%$, at 26 versus $36.5 \%$, the BC group survival in the present series was clearly poorer than that reported in the same study [16]. KIM et al. [16] observed no difference in stages
I and II, in contrast with the present study, in which the actuarial 5-yr survival rate was $44 \%$ in the NSCLC group and $27 \%$ in the $B C$ group (log rank $p=0.01$ ). Interestingly, this difference was maintained when BC and SCC were compared with regard to survival in stages I (33 versus 51\%; log rank $p=0.02$ ) and the median survival of 36 months for BC was not reached at 5 yrs for SCC. In addition, in order to avoid bias related to occult concomitant medical conditions, comorbidities were scored and added to the analysis. Basaloid pattern confirmed its poor prognostic significance in uni- and multivariate analyses in the complete group of patients, and especially in earlier stage tumours, such as resected stage I. In addition, patients with more advanced stages (IIIB and IV) were more frequent in the BC group.

Since an excess of post-operative deaths, even if statistically nonsignificant, was observed in the $\mathrm{BC}$ group, the current authors wondered whether their prognostic difference was biased by surgical mortality, a point raised by the peer review of their original publication [1]. In the current analysis, the authors confirm the prognostic impact of basaloid histology, either in the complete group of patients or in the group of patients alive after the 30 days following surgical resection. However, as carcinoma with a basaloid pattern was frequently associated with CIS, which could be responsible for a more aggressive surgery, the implication of CIS in this increase of peri-operative deaths has been sought. No differences were found between BC and NSCLC or SCC with regard to the extent of surgical resection (lobectomy versus pneumonectomy). However, BC patients were observed to be heavier smokers, which could explain the excess of concomitant CIS diagnosed in this population $(p<0.0001)$ and evokes its histogenesis from proliferative bronchial basal stem cells.

Surgical therapy might not be sufficient or curative for basaloid carcinomas, as inferred by their poor prognosis after surgery at stage I. Adjuvant chemotherapy is presently considered as a standard of care, since beneficial effects have been demonstrated in stages IB-IIIA nonsmall cell lung carcinoma [20]. However, some controversies still exist over the impact of adjuvant chemotherapy in stage IB [21, 22], which requires additional pathological and immunohistochemical criteria to identify those patients who could benefit most from adjuvant treatments. Since carcinoma with a basaloid pattern remains a rare tumour, patients should be included in prospective large-scale and multicentre chemotherapy regimens. For instance, stratification of patients could be based on the expression of biomarkers of drug-associated resistance.

TABLE 4 Variables in a multivariate Cox model in stage I patients 
Indeed, substantial survival benefits from cisplatin-based chemotherapy in patients with ERCC1 and P27 nonsmall cell lung carcinoma-negative tumours have recently been demonstrated $[23,24]$. Future studies will require a better definition of the basaloid carcinoma entity at the histological level to adequately include patients in randomised adjuvant trials.

\section{ACKNOWLEDGEMENTS}

The authors would like to thank G. Butt (Faculté de Medicine, Université Joseph Fourrier, La Tronche, France) for the careful revision and correction of this manuscript.

\section{REFERENCES}

1 Brambilla E, Moro D, Veale D, et al. Basal cell (basaloid) carcinoma of the lung: a new morphologic and phenotypic entity with separate prognostic significance. Human Pathol 1992; 23: 993-1003.

2 Sarbia M, Verreet P, Bittinger F, et al. Basaloid squamous cell carcinoma of the esophagus: diagnosis and prognosis. Cancer 1997; 79: 1871-1878.

3 Kawashima O, Kamiyoshihara M, Sakata S, Kurihara T, Ishikawa S, Morishita Y. Basaloid carcinoma of the thymus. Ann Thorac Surg 1999; 68: 1863-1865.

4 Andreadis D, Nomikos A, Epivatianos A, Poulopoulos A, Barbatis C. Basaloid squamous cell carcinoma versus basal cell adenocarcinoma of the oral cavity. Pathology 2005; 37: 560-563.

5 Teramoto N, Nishimura R, Saeki T, Nogawa T, Hiura M. Adenoid basal carcinoma of the uterine cervix: report of two cases with reference to adenosquamous carcinoma. Pathol Int 2005; 55: 445-452.

6 Wain SL, Kier R, Vollmer RT, Bossen EH. Basaloidsquamous carcinoma of the tongue, hypopharynx, and larynx: report of 10 cases. Hum Pathol 1986; 17: 1158-1166.

7 Moro D, Brichon PY, Brambilla E, Veale D, Labat F, Brambilla C. Basaloid bronchial carcinoma: a histological group with a poor prognosis. Cancer 1994; 73: 2734-2739.

8 Sturm N, Lantuejoul S, Laverriere MH, et al. Thyroid transcription factor 1 and cytokeratins 1, 5, 10, 14 (34ßE12) expression in basaloid and large-cell neuroendocrine carcinomas of the lung. Hum Pathol 2001; 32: 918-925.

9 Brambilla E. Basaloid carcinoma. In: Brambilla C, Brambilla E, eds. Lung Tumors: Fundamental Biology and Clinical Management. New York, Marcel Dekker, 1998; pp. 55-68.

10 Travis WD, Colby TV, Corrin B, et al. Histological typing of lung and pleural tumors. 3rd Edn. Geneva, World Health Organization, 1999.

11 Travis WD, Brambilla E, Müller-Hermelink HK, Harris CC. Pathology and Genetics: Tumours of the Lung, Pleura,
Thymus and Heart. Lyon, International Agency for Research on Cancer, 2004.

12 Noguchi M, Morikawa A, Kawasaki M, et al. Small adenocarcinoma of the lung. Histologic characteristics and prognosis. Cancer 1995; 75: 2844-2852.

13 Travis WD, Linnoila RI, Tsokos MG, et al. Neuroendocrine tumors of the lung with proposed criteria for large-cell neuroendocrine carcinoma. An ultrastructural, immunohistochemical, and flow cytometric study of 35 cases. Am J Surg Pathol 1991; 15: 529-553.

14 Travis WD, Rush W, Flieder DB, et al. Survival analysis of 200 pulmonary neuroendocrine tumors with clarification of criteria for atypical carcinoid and its separation from typical carcinoid. Am J Surg Pathol 1998; 22: 934-944.

15 Rossi G, Cavazza A, Sturm N, et al. Pulmonary carcinomas with pleomorphic, sarcomatoid, or sarcomatous elements: a clinicopathologic and immunohistochemical study of 75 cases. Am J Surg Pathol 2003; 27: 311-324.

16 Kim DJ, Kim KD, Shin DH, Ro JY, Chung KY. Basaloid carcinoma of the lung: a really dismal histologic variant? Ann Thorac Surg 2003; 76: 1833-1837.

17 Mountain CF. Revisions in the International System for Staging Lung Cancer. Chest 1997; 111: 1710-1777.

18 Charlson ME, Pompei P, Ales KL, MacKenzie CR. A new method of classifying prognostic comorbidity in longitudinal studies: development and validation. J Chronic Dis 1987; 40: 373-383.

19 Schemper M, Smith T. A note on quantifying follow-up in studies of failure time. Controlled Clin Trials 1996; 17: 343-346.

20 Arriaguda R, Bergman B, Dunant A, et al. Cisplatin-based adjuvant chemotherapy in patients with completely resected non-small-cell lung cancer. N Engl J Med 2004; 350: 351-360.

21 Douillard JY, Rosell R, De Lena M, et al. Adjuvant vinorelbine plus cisplatin versus observation in patients with completely resected stage IB-IIIA non-small-cell lung cancer (Adjuvant Navelbine International Trialist Association [ANITA]): a randomised controlled trial. Lancet Oncol 2006; 7: 719-727.

22 Winton T, Livingston R, Johnson D, et al. Vinorelbine plus cisplatin versus observation in resected non-small-cell lung cancer. N Engl J Med 2005; 352: 2589-2597.

23 Olaussen KA, Dunant A, Fouret P, et al. DNA repair by ERCC1 in non-small-cell lung cancer and cisplatin-based adjuvant chemotherapy. N Engl J Med 2006; 355: 983-991.

24 Filipits M, Pirker R, Dunant A, et al. Cell cycle regulators and outcome of adjuvant cisplatin-based chemotherapy in completely resected non-small-cell lung cancer: the International Adjuvant Lung Cancer Trial Biologic Program. J Clin Oncol 2007; 25: 2735-2740. 